\title{
BMJ Open Effectiveness of interventions for the prevention of occupational violence against professionals in health services: a protocol for a systematic review
}

\author{
Caroline Vieira Cláudio Okubo (D) , ${ }^{1,2}$ Renata Cristina Campos Pereira Silveira, ${ }^{3}$ \\ Maria José Quina Galdino, ${ }^{4}$ Daiane Rubinato Fernandes, ${ }^{3}$ \\ Aline Aparecida Oliveira Moreira, ${ }^{1}$ Júlia Trevisan Martins ${ }^{5}$
}

To cite: Okubo CVC, Silveira RCCP, Galdino MJQ, et al. Effectiveness of interventions for the prevention of occupational violence against professionals in health services: a protocol for a systematic review. BMJ Open 2020;10:e036558. doi:10.1136/ bmjopen-2019-036558

- Prepublication history for this paper is available online. To view these files, please visit the journal online (http://dx.doi org/10.1136/bmjopen-2019036558).

Received 19 December 2019 Revised 08 August 2020 Accepted 10 August 2020

Check for updates

(C) Author(s) (or their employer(s)) 2020. Re-use permitted under CC BY-NC. No commercial re-use. See rights and permissions. Published by BMJ.

For numbered affiliations see end of article.

Correspondence to

Caroline Vieira Cláudio Okubo; caroline.vieirac@gmail.com

\section{ABSTRACT}

Introduction Occupational violence affects several categories of workers; however, the health sector category has been considered at a high risk, exposing workers to physical and psychological abuse. Thus, occupational violence has decreased the quality of care in health service. This review aims to evaluate the effectiveness of interventions for the prevention and reduction of occupational violence against health professionals.

Methods and analysis This protocol is consistent with the Preferred Reporting Items for Systematic Review and Meta-Analysis Protocols. Searches will be conducted in PubMed, Embase, Cochrane Library, LILACS, Web of Science, Scopus, CINAHL and LIVIVO along with a comprehensive review of grey literature. The search will be conducted on August 1 st 2020, without language and time restrictions. Following the eligibility criteria, two independent reviewers will select the titles and abstracts and subsequently screen the full articles. If necessary, a third reviewer will assess any disagreements. All references will be imported into EndNote, and any duplicates will be removed. The data will be extracted using an extraction-based form from Cochrane. Statistical analyses will be performed using the software Cochrane Review Manager, and a meta-analysis will be performed if possible for the statistical combination of at least two studies. The risk of bias of the randomised clinical trials will be evaluated by the Risk of Bias tool from Cochrane, and the risk of bias of the non-randomised intervention studies will be evaluated using the Downs and Black scale. The quality of the evidence and strength of the classification recommendations will be assessed by the Grading of Recommendations, Assessment, Development and Evaluation.

Ethics and dissemination This review will not evaluate individual patient information and therefore does not require ethical approval. The results will be disseminated through publications in peer-reviewed journals, presentations at conferences and the doctoral thesis of the leading author.

PROSPERO registration number CRD42018111383.

\section{Strengths and limitations of this study}

- To the best of our knowledge, this systematic review will be the first to evaluate the interventions used to prevent occupational violence against health professionals.

- This manuscript design adheres to all relevant guidelines for systematic reviews.

- The databases will be searched without time and language restrictions.

- A limitation of this study may be that high heterogeneity among the included studies may affect the quality of the evidence due to variations in study designs and professional characteristics among the included studies.

\section{INTRODUCTION}

Occupational violence constitutes a major public health problem worldwide and has aroused the interest of researchers since it represents a major source of inequality, alienation and conflict in the workplace. ${ }^{12}$

The International Labour Organization (ILO) defines occupational violence as any action or behaviour that deviates from the established conduct at work in which a person is assaulted and threatened in their workplace or as a direct result of their work. ${ }^{3}$

This violence can occur internally between workers in a sector or institution, including managers and supervisors, or externally between health workers and any other person present in the workplace who is not a health professional, such as a patient or caregiver. ${ }^{3}$

Violence in the workplace can be physical (murder, attack, spitting, kicking, beating or rape) or psychological (mobbing, bullying, inducing fear, threatening, abusive behaviour or harassment), mainly affects the health sector and is considered a high risk for workers due to the characteristics of the 
services provided and the current working environment, especially considering the long working hours, shift work, and labour reforms. As negative consequences, there is a decrease in workers and the quality of services provided as some workers have chosen to leave the profession in response to violence. ${ }^{1}$

The trend of violence at work has continuously increased, and since 2002, an international response began when the ILO, the International Council of Nurses, the World Health Organization (WHO) and the International Civil Service Federation issued guidelines for addressing occupational violence in the health sector, including an analysis of the workplace, prevention and training concerning health and safety, thus offering an effective approach to reducing or eliminating the risks to which workers are exposed. ${ }^{45}$

Violence in the workplace affects all countries and services, and approximately $4 \%$ of the global population of workers have experienced physical violence. The health sector accounts for $25 \%$ of all violence at work, resulting in decreased productivity in institutions, absenteeism, stress and even more violence. Nurses and doctors are at the top of the list of occupations with high levels of violence; however, nurses are three times more exposed than workers in any other profession. ${ }^{5}$

International studies and guidelines have identified that occupational violence, including both physical or psychological, mainly occurs in the emergency department, home-care and geriatric units and centers on women. ${ }^{467}$

Studies reinforce that the prevalence of occupational violence targeting health service workers is high, reaching more than half of these workers, mainly doctors and nurses in emergency departments, with a predominance of verbal abuse and physical violence. ${ }^{89}$ A study conducted with 378 nurses in emergency departments found that more than $90 \%$ of this category of workers suffered violence at work in recent years and that preventive measures were likely not effective,${ }^{10}$ exposing professionals to this occupational risk.

Systematic reviews have analysed the prevalence, incidence, ${ }^{11} 12$ antecedents and consequences ${ }^{13}{ }^{14}$ of occupational violence, especially in emergency services. ${ }^{15}$ However, few reviews involving meta-analyses addressed the effectiveness of interventions for the prevention of occupational violence against professionals in health services.

The preventive measures used to combat occupational violence that have been evaluated include educational training conducted with the hospital nursing team, which was shown to decrease the incidence rates of aggression. ${ }^{16}$ Another study evaluated an electronic program addressing an algorithm of the waiting time of patients at a university hospital and noted a decrease in the incidence rate of violence among the health team. ${ }^{17}$

Systematic reviews ${ }^{18}{ }^{19}$ have also sought to answer the question of the effectiveness of interventions carried out to prevent violence against health workers; however, authors $^{18} 19$ have found low to moderate evidence regarding prevention programs carried out in emergency departments. One study ${ }^{18}$ evaluated the evidence according to the classification proposed by Oxford, ${ }^{20}$ and another study ${ }^{19}$ evaluated the evidence but did not present the tools used for the measurement. Therefore, this review is justified to seek studies with high scientific evidence. ${ }^{21} 22$

In view of the above, the following questions emerge: Which interventions prevent occupational violence against health professionals? Which interventions effectively reduce occupational violence against professionals in health services? Thus, the general objective of this review is to evaluate the effectiveness of interventions for the prevention and reduction of occupational violence against health professionals.

\section{METHODS AND ANALYSIS}

\section{Review design}

This systematic review protocol is consistent with the checklist Preferred Reporting Items for Systematic Review and Meta-Analysis Protocols (PRISMA-P) (online supplemental file 1). ${ }^{23}{ }^{24}$ This systematic review will also be conducted according to the PRISMA guidelines. ${ }^{25}$ The research question was developed based on the acronym PICO (Population, Intervention, Comparison or Control, and Outcome), that is, 'which interventions are effective in preventing and reducing occupational violence against professionals in health services?', described in detail below.

\section{Inclusion and exclusion criteria \\ Types of participants}

Health professionals in health services, including doctors, nurses, physical therapists and paramedical practitioners, among others, in addition to health associate professionals, such as nursing associate professional, medical assistants, ambulance workers and community health workers; and personal care workers in health services and health management and support personnel, such as nursing aides, medical imaging assistants, medical secretaries, ambulance drivers, clinical psychologists, social workers and cleaners will be included. These criteria are based on the WHO literature. ${ }^{26}$

\section{Intervention type and exposure}

Any type of intervention used to prevent occupational violence among health professionals, health associate professionals and personal care workers in health services exposed to occupational violence will be eligible.

\section{Control interventions}

The control interventions will include standard intervention, usual interventions, or no interventions.

\section{Outcome measures}

The outcome measures include the prevention or reduction of occupational violence (physical and psychological) 
against health professionals, health associate professionals, personal care workers and health management and support personnel in health services.

\section{Types of studies}

We will include randomised and non-randomised clinical trials; clinical trials adopting quasi-randomised, controlled, randomised, experimental or quasiexperimental designs of the before and after type and cross-sectional, longitudinal cohort studies and other observational studies that provide quantitative estimates and interventions results.

\section{Exclusion criteria}

Studies that do not meet the eligibility criteria and do not answer the PICO research question', will be excluded. Studies conducted in medical offices or at home with outcomes other than the prevention and reduction of violence and qualitative research, for example, observational studies, will also be excluded.

\section{Search strategy}

The objective of this review is to identify eligible studies regardless of language or publication status (published, unpublished, in press or in progress) in existence prior to August 1 st 2020, but we will update the search as necessary to cover all articles to the date of publication of the data. The search will be conducted from the inception of each database on August 1 st 2020, without language and time restrictions. The overall study will be conducted until December 10 th 2020.

Searches will be conducted in the following electronic databases: PubMed, Embase, the Cochrane Library, LILACS, Web of Science, Scopus, CINAHL and LIVIVO. The complete search strategy using the PubMed database is shown in online supplemental table 1 . This strategy will be modified appropriately for other databases.

The electronic records of completed or ongoing clinical trials will be identified from the following databases: Clinical Trials of the United States of America (USA), International Clinical Trials Platform Records of the WHO and the Brazilian Registry of Clinical Trials (Rebec) of the Ministry of Health of Brazil. Dissertations and theses will also be included through a search using ProQuest and banked theses and dissertations at University of São Paulo (USP, Brazil). We will also use OpenGrey and Google Scholar to search for grey literature.

After the inclusion of eligible studies is accomplished, we will manually search the lists of articles included in the review references. If necessary, the corresponding author of the study will be contacted via email to request information regarding the studies.

\section{Data collection and analysis}

\section{Selection of studies}

The selection of the studies will occur in two stages. First, two independent reviewers will review the titles and abstracts of all identified electronic database articles. If disagreement occurs, a third author will be involved.
Second, the same selection criteria will be applied to full text articles to confirm their eligibility. The same two reviewers will independently participate in this step. The selected articles will be read in their entirety. Any disagreement at any stage will be settled by mutual agreement among the three reviewers.

All references obtained through this process will be imported into EndNote, and duplicates will be removed. After their removal, the studies will be inserted into the electronic platform Rayyan.

\section{Extraction and data management}

Two authors will extract the data independently using a form based on the data extraction form of Cochrane. ${ }^{27}$ The extracted data will include the following: information regarding the publication, such as the authors, country, journal name and year of publication; methodological study design and quality (such as allocating in a blinded randomised clinical trial); participants, including the sample size and participant characteristics (sex, age, etc); type of intervention and outcome data. Disagreements regarding the extracted data will be resolved by consensus, and a third reviewer will be consulted if any discrepancy persists. The authors of the studies will be contacted to provide information in cases of missing data or when the presented data are unclear.

\section{Data analysis}

The software Cochrane's Review Manager (V.5.3) will be used to perform the statistical analyses. A meta-analysis will be performed if possible for statistical combine at least two studies.

Notably, in this review, the interventions will be considered effective if there is prevention or reduction of occupational violence. If possible, we will conduct a metaanalysis with a dichotomous outcome, that is, we will describe whether there was prevention of and a decrease in occupational violence against health professionals. If it is not possible to perform the analysis with dichotomous data, we will perform a meta-analysis with continuous data. Moreover, a rigorous narrative analysis will be performed. We will not perform a cost-effectiveness analysis of the interventions.

For dichotomous data, we will use the Risk Ratio (RR), Odds Ratio (OR) and/or risk difference with a Confidence Interval (CI) of 95\%. For continuous data, the average difference will be used if the results are measured in the same manner. We will also use the standardised mean difference to combine studies that measure the same outcome but use different methods. For continuous data, we will also use a $95 \% \mathrm{CI}^{28}$

Heterogeneity will be assessed by visual inspection of a forest plot, $\chi^{2}$ tests and/or the $\mathrm{I}^{2}$ test statistic; if the $\mathrm{I}^{2}$ test statistic is less than $75 \%$, the data may be combined. If possible, the methodological and clinical heterogeneity will also be evaluated. ${ }^{28}$

A random-effect model will be used when heterogeneity exists among the studies, while a fixed-effect model 
will be used if no heterogeneity exists among the studies or $\mathrm{I}^{2}$ is less than $25 \%$.

If possible, a subgroup analysis will be performed to identify potential effect modifiers, such as the characteristics of the participants, including the professional category (nurse or doctor), workplace (hospital, health facility, emergency units and long-term care facilities) and intervention characteristics, such as the type and duration of the intervention.

A sensitivity analysis will also be performed to assess the impact of studies with a high risk of bias. We will discuss whether studies with a lower quality should be excluded from the analysis based on their sample size, evidence of their strength and the influence of the size grouped effect. $^{28}$

\section{Risk of bias and reporting of the study quality}

Two authors will independently assess the risk of bias of the included studies using the 'Risk of Bias' tool of Cochrane for randomised controlled trials. The following areas will be evaluated: generation of a random sequence, allocation concealment, blinding of the participants and staff, blinding of the assessors of outcomes, incomplete data outcomes, selective reporting and other biases. Each criterion will be judged explicitly as follows: low risk, high risk or uncertain risk of bias. ${ }^{28}$

In the case of intervention studies without randomisation, we will use the Downs and Black scale to assess the risk of bias while covering the following areas: reporting, external validity, internal validity (bias), internal validity (confounding) and statistical power. ${ }^{29}$ Disagreements regarding the risk of bias will be solved by consensus between the two reviewers.

The observational studies included in this review will also have their risk of bias assessed using the NewcastleOttawa scale, which includes the following items: selection, comparison, exposure or outcome. ${ }^{30}$

\section{Evidence of the quality}

The quality of the evidence and the strength of the classification of recommendations will be evaluated using Grades of Recommendation, Assessment, Development and Evaluation (GRADE) with the help of the software GRADEpro. ${ }^{212231}$ The criteria used for this evaluation will be the risk of bias, inconsistency of the results, the direction of the evidence, inaccuracy of the results, imprecision of guidelines, publication bias and other factors as appropriate. ${ }^{21} 22$

The quality of the evidence will be characterised as high (there is great confidence that the true effect is close to the estimate of the effect), moderate (there is moderate confidence in the estimate of the effect), low (confidence in the estimate of the effect is limited) or very low (there is very limited confidence in the estimate of the effect).$^{2122}$

\section{Patient and public involvement}

Patients and the public will not be directly involved in the performance of this systematic review. However, through publications in journals and presentations at national and international conferences, we will attempt to make the results available to the maximum number of people possible, including health professionals.

\section{Ethics and dissemination}

In accordance with the guidelines of the PRISMA-P, ${ }^{23}{ }^{24}$ this systematic review protocol was registered in the International Prospective Register of Systematic Reviews (PROSPERO) on October 24 th 2018. After registration, this review was performed and included the databases Embase and LIVIVO, non-randomised controlled trials; quasi-randomised, controlled, randomised, experimental and quasi-experimental studies with a before and after design and cross-sectional, longitudinal cohort and other observational studies.

The dissemination of this review will be accomplished through publications in peer-reviewed journals, presentations at national and international conferences and the doctoral dissertation of the leading author.

\section{Amendments to the protocol}

If there is a need to amend this protocol, the date of each amendment and the reason for the change will be described.

\section{Author affiliations}

${ }^{1} \mathrm{PhD}$ student in Nursing, State University of Londrina, Londrina, Paraná, Brazil ${ }^{2}$ Nurse, Federal University of Paraná Clinics Hospital, Curitiba, Paraná, Brazil

${ }^{3}$ University of São Paulo at Ribeirão Preto College of Nursing, Ribeirão Preto, São Paulo, Brazil

${ }^{4}$ Department of Nursing, Northern State University of Paraná, Bandeirantes, Paraná, Brazil

${ }^{5}$ Department of Nursing, State University of Londrina, Londrina, PR, Brazil

Acknowledgements The authors would wish to thank the State University of Londrina for support and AJE (American Journal Experts) for editing the English text of the draft of this manuscript.

Contributors CVCO, JTM and RCCPS developed the systematic review protocol. CVCO, MJQG, DRF and AAOM wrote this protocol. RCCPS reviewed this protocol. CVCO, MJQG and AAOM contributed to the development of the data selection criteria, assessment of the risk of bias and extraction and analysis of the data. CVCO, JTM, RCCPS and DRF developed the search strategy. CVCO, JTM, RCCPS, MJQG, DRF and AAOM read and approved the final version. CVCO is the guarantor of this systematic review.

Funding The authors have not declared a specific grant for this research from any funding agency in the public, commercial or not-for-profit sectors.

Competing interests None declared.

Patient and public involvement Patients and/or the public were not involved in the design, or conduct, or reporting, or dissemination plans of this research.

Patient consent for publication Not required.

Provenance and peer review Not commissioned; externally peer reviewed.

Open access This is an open access article distributed in accordance with the Creative Commons Attribution Non Commercial (CC BY-NC 4.0) license, which permits others to distribute, remix, adapt, build upon this work noncommercially, and license their derivative works on different terms, provided the original work is properly cited, appropriate credit is given, any changes made indicated, and the use is non-commercial. See: http://creativecommons.org/ licenses/by-nc/4.0/.

ORCID iD

Caroline Vieira Cláudio Okubo http://orcid.org/0000-0001-8625-8667 


\section{REFERENCES}

1 International Labor Organization. Framework guidelines for addressing workplace violence in the health sector - the training manual. The Training manual. Geneva: Switzerland, 2005.

2 International Organización del Trabajo. Consejo Internacional de Enfermeras, world La Salud Organización, Servicios Públicos international. Geneva: ILO: Directrices March to face her occupational violence en el sector de la Salud, 2002. https://apps. who.int/iris/handle/10665/44072

3 International Labor Organization. Code of practice on workplace violence in services sectors and measures to combat this phenomenon. Geneva: International Labor Organization, 2003.

4 Occupational Safety and Health Act (OSHA). Guidelines for preventing workplace violence for healthcare and social service workers. Washington: OSHA, 2016.

5 Martino D V. Workplace violence in the health care sector relationship between work stress and workplace violence in the health sector. Available: http://bit.ly/1Szm14B [Accessed 10 Jun 2020].

6 Mishra S, Chopra D, Jauhari N, et al. Violence against health care workers: a provider's (staff nurse) perspective. Int J Community Med Public Health 2018;5:4140-8.

7 Sisawo EJ, Ouédraogo SYYA, Huang S-L. Workplace violence against nurses in the Gambia: mixed methods design. BMC Health Serv Res 2017;17:311.

8 Paravic-Klijn T, Burgos-Moreno M. Prevalencia de violencia física, abuso verbal y factores asociados en trabajadores/as de servicios de emergencia en establecimientos de salud públicos y privados [Verbal and physical abuse towards health care workers in emergency services]. Rev Med Chil 2018;146:727-36.

9 Liu J, Gan Y, Jiang H, et al. Prevalence of workplace violence against healthcare workers: a systematic review and meta-analysis. Occup Environ Med 2019;76:927-37.

10 Lee H-L, Han C-Y, Redley B, et al. Workplace violence against emergency nurses in Taiwan: a cross-sectional study. J Emerg Nurs 2020;46:66-71.

11 Lu L, Dong M, Wang S-B, et al. Prevalence of workplace violence against health-care professionals in China: a comprehensive meta-analysis of observational surveys. Trauma Violence Abuse 2020;21:498-509.

12 Azami M, Moslemirad M, YektaKooshali MH, et al. Workplace violence against Iranian nurses: a systematic review and metaanalysis. Violence Vict 2018;33:1148-75.

13 Nowrouzi-Kia B, Isidro R, Chai E, et al. Antecedent factors in different types of workplace violence against nurses: a systematic review. Aggress Violent Behav 2019;44:1-7.

14 Lanctôt N, Guay S. The aftermath of workplace violence among healthcare workers: a systematic literature review of the consequences. Aggress Violent Behav 2014;19:492-501.

15 Maguire BJ, O'Meara P, O'Neill BJ, et al. Violence against emergency medical services personnel: a systematic review of the literature. Am $J$ Ind Med 2018;61:167-80.
16 Lakatos BE, Mitchell MT, Askari R, et al. An interdisciplinary clinical approach for workplace violence prevention and injury reduction in the General Hospital setting: S.A.F.E. response. J Am Psychiatr Nurses Assoc 2019;25:280-8.

17 Touzet S, Occelli P, Denis A, et al. Impact of a comprehensive prevention programme aimed at reducing incivility and verbal violence against healthcare workers in a French ophthalmic emergency department: an interrupted time-series study. BMJ Open 2019;9:e031054.

18 Ramacciati N, Ceccagnoli A, Addey B, et al. Interventions to reduce the risk of violence toward emergency department staff: current approaches. Open Access Emerg Med 2016;8:17-27.

19 Raveel A, Schoenmakers B. Interventions to prevent aggression against doctors: a systematic review. BMJ Open 2019;9:e028465.

20 Howick J, Chalmers I O. Levels of evidence Working Group. levels of evidence 2 the Oxford, 2011. Available: http://www.cebm.net/index. aspx?o=1025 [Accessed 10 Jun 2020].

21 Schünemann HJ, Brożek J, Guyatt G, et al. GRADE Handbook for quality grading of evidence and strength of recommendations. GRADE: The GRADE Working Group, 2013. Available: https://gdt. gradepro.org/app/handbook/handbook.html [Accessed 08 Jun 2019].

22 Balshem H, Helfand M, Schünemann HJ, et al. Grade guidelines: 3 rating the quality of evidence. J Clin Epidemiol 2011;64:401-6.

23 Moher D, Stewart L, Shekelle P. Implementing PRISMA-P: recommendations for prospective authors. Syst Rev 2016;5:15.

24 Shamseer L, Moher D, Clarke M, et al. Preferred reporting items for systematic review and meta-analysis protocols (PRISMA-P) 2015 elaboration and explanation. BMJ 2015;349:7647.

25 Moher D, Liberati A, Tetzlaff J, et al. Preferred reporting items for systematic reviews and meta-analyses: the PRISMA statement. PLoS Med 2009;6:E1000097.

26 World Health Organization. Classification of health workforce statistics. Available: https://www.who.int/hrh/statistics/Health_ workers_classification.pdf?ua=1 [Accessed 11 Jun 2020].

27 Cochrane. Data extraction forms, 2019. Available: https://dplp. cochrane.org/data-extraction-forms [Accessed $10 \mathrm{Jul} 2019$ ].

28 Higgins JPT, Green S. Cochrane Handbook for systematic reviews of interventions version 5.1.0. The Cochrane collaboration, 2011. Available: www.handbook.cochrane.org [Accessed 2 Jul 2019].

29 Downs HS, Black N. The feasibility of creating the checklist for the methodological assessment of the quality both of non-randomized and randomized studies of health care interventions. J Epidemiol Community Health 1998;52:377-84.

30 Wells GA, Shea B D O'Connell. The Newcastle-Ottawa scale (NOS) for assessing the quality of nonrandomised studies in meta-analyzes, 2019. Available: http://www.ohri.ca/programs/clinical_epidemiology/ oxford.asp [Accessed 12 Jul 2019].

31 GRADEpro GDT: GRADEpro Guideline Development Tool [Software]. McMaster University, 2015. Available: https://gradepro.org./ [Accessed 23 Jun 2019]. 